\title{
Variación de la comunidad de abejas de las orquídeas (Hymenoptera: Apidae) en tres ambientes perturbados del piedemonte llanero colombiano
}

\author{
Alejandro Parra-H. ${ }^{1}$ \& Guiomar Nates-Parra ${ }^{2}$ \\ Laboratorio de Investigaciones en Abejas LABUN, Departamento de Biología, Universidad Nacional de Colombia, \\ Bogotá, Carrera 30 N 45-03; 1. varnishpt@yahoo.com, 2.mgnatesp@unal.edu.co
}

Recibido 08-VI-2006. Corregido 15-I-2007. Aceptado 07-V-2007.

\begin{abstract}
Variation of the orchid bees community (Hymenoptera: Apidae) in three altered habitats of the Colombian "Ilano" piedmont. Orchid bees subsist in vast tropical forest areas because they maintain close relationships with particular plant species in diverse micro-habitats. Based on the relationships among the environment and biological features (food preference, morphologic and ethologic diversity), it is possible to determine habitat quality using the euglossine array. This work proposes the use of this ecological information, in addition to diversity indices, for the evaluation of environmental quality. Fifteen localities in three landscape types (urban, rural and conserved) were sampled in the eastern llanos foothill (Meta, Colombia), between March and December of 2003 using entomological nets, and Cineol and Metil Salicylate as baits. Of the 26 species known to occur in the area, 17 were registered. Eulaema nigrita was the most frequent, while E. speciosa E. bombiformis, Euglossa magnipes, E. cybelia, E. heterosticta, E. singularis and Exaerete frontalis were mostly found in habitats rated "good to acceptable". The vegetation composition and proximity of forest fragments seem to favor some species in disturbed habitats. Relative diversity of bee body shapes and sizes is proportional to habitat quality. Rev. Biol. Trop. 55 (3-4): 931-941. Epub 2007 December, 28.
\end{abstract}

Key words: Colombian eastern llanos piedmont, communities, conservation, diversity, euglossine bees.

El conocimiento sobre las abejas de las orquídeas (Apidae: Euglossini) se incrementó notablemente con el descubrimiento y posterior uso de atrayentes aromáticos (Dodson et al. 1969), lo cual ha permitido conocer información de este grupo de organismos, como por ejemplo, sus posibles agrupamientos dentro de un hábitat (Dressler 1969) y su diversidad taxonómica (Dressler 1982, Roubik 1989, Cameron 2004).

Este grupo de abejas de lengua larga y amplios ámbitos de vuelo (Dudley 1995), juegan un papel crítico en la estabilidad de diversas familias de plantas de los bosques neotropicales, ya que les prestan el servicio de polinización (Dressler 1968, Silva y Rebêlo 2002, Otero y Sandino 2003, Roubik y Hanson
2004). Ocupan diversos hábitat de extensas áreas de bosques neotropicales debido a que pueden encontrar en estos los recursos necesarios para cubrir sus requerimientos de nidificación y alimentación (Roubik y Hanson 2004). A la vez la capacidad de vuelo de las abejas euglosinas, implicada en la eficiencia de polinización de muchas de estas familias de plantas, se ve afectada por diversos factores bióticos como es la oferta de recursos energéticos que, dependiendo de su concentración, serán más o menos atractivos (Kato et al. 1992). La capacidad de mantenimiento de los euglosinos (estrechamente relacionada con las distancias que recorren y su comportamiento), estaría determinada por la calidad y características físicas y ecológicas del hábitat en particular 
(Roubik 1989, Silva y Rebêlo 2002, Otero y Sandino 2003, Roubik y Hanson 2004, Parra-H et al. 2005), por lo cual es de especial importancia el grado de conservación de los bosques en su relación con la comunidad de abejas de las orquídeas (Roubik y Hanson 2004, Parra-H y Ospina-Torres, pers. obs).

Dada la gran amenaza que representa la fragmentación para la manutención de poblaciones naturales de estos organismos, se han realizado diversos ensayos en los que se ha evidenciado cómo el efecto de la presión humana sobre el medio, especialmente la fragmentación de las coberturas vegetales, desequilibra la composición natural de las poblaciones de euglosinos y desplaza a las abejas de las orquídeas a los bordes de los fragmentos, que por el tipo de vegetación que aparece, ofrece más recursos energéticos y amenaza con desestabilizar las dinámicas de polinización de especies vegetales en las áreas al interior de los bosques (Powell y Powell 1987).

Los insectos en general responden a los cambios del medio en diversos ámbitos de su vida, siendo la agricultura (Magagula 2003) y la ganadería (Márquez 2001) los factores y las causas más evidentes de la perturbación de los ecosistemas.

Este trabajo muestra el comportamiento en la distribución de las especies de euglosinos, en tres ambientes con distinto grado de perturbación humana en el piedemonte llanero colombiano durante un año. A la vez propone una interpretación sobre el uso de ésta información para caracterizar cualitativamente el grado de perturbación y calidad de hábitat, según características biológicas y ecológicas de esta tribu, como sugiere Bonilla-Gómez (1997).

\section{MATERIALES Y MÉTODOS}

Entre marzo y diciembre de 2003 se muestrearon mensualmente tres municipios en el piedemonte llanero del oriente colombiano: Acacías $\left(4^{\circ} 00^{\prime} \mathrm{N}-73^{\circ} 46^{\prime} \mathrm{W}\right)$ Cumaral $\left(4^{\circ} 17^{\prime}\right.$ N- $73^{\circ} 30^{\prime}$ W) y Villavicencio $\left(4^{\circ} 09^{\prime}\right.$ N- $73^{\circ} 39^{\prime}$ W). Dentro de cada municipio se seleccionaron localidades con diferentes niveles de presión antrópica: urbano (cabeceras municipales con una población hasta de 400000 habitantes sin coberturas de vegetación nativa), rural (zonas aledañas a los cascos urbanos o áreas con explotación agrícola con menos de 130 000 habitantes, con fragmentos de vegetación boscosa en promedio de $2 \mathrm{Ha}$.) y ambiente relativamente conservado (áreas con cobertura vegetal contigua de más de $20 \mathrm{Ha}$., sin asentamientos humanos).

El área de estudio corresponde a la zona de vida bosque muy húmedo tropical (bmh-T) (Rangel-Ch y Aguilar-P 1995). La vegetación circundante a las áreas de bosque, en la región, históricamente ha estado representada por sabanas multifloras con bosques relictuales, sabanas húmedas con coberturas leñosas achaparradas, bosques de galería asociados a recursos hídricos provenientes de los andes y sabanas inundables (Blyndenstein 1967). La temperatura media es de $26{ }^{\circ} \mathrm{C}$, la precipitación anual es de $3200-4500 \mathrm{~mm}$., la humedad relativa es de $84 \%$ en época lluviosa y $63 \%$ en época seca (Rodríguez-C 2005). La región se usa extensivamente en ganadería, a la vez que se cultiva intensamente palma africana, soya, arroz y cítricos, lo cual afecta principalmente coberturas de vegetación nativa y suelos (CORPOMACARENA 2004).

Los muestreos consistieron en capturas directas con red entomológica en cada una de las localidades escogidas, a la vez que se realizaron capturas con los atrayentes aromáticos más generalistas para euglosinos: Cineol y Metil Salicilato (Dressler 1969). Estos se realizaron durante períodos de $6 \mathrm{~h}$ aproximadamente, incluyendo los picos de actividad de estas abejas (08:00 y 14:00). El material colectado fue ingresado a la colección del Laboratorio de Investigaciones en Abejas LABUN, Departamento de Biología, Universidad Nacional de Colombia y se determinó hasta especie usando las claves de Bonilla-Gómez y Nates-Parra (1992).

Se determinó la diversidad de especies de euglosinos para las localidades de los municipios muestreados por medio del índice $\mathrm{H}$ de 
MacArthur y MacArthur (1961). Además, se realizó una comparación de las comunidades de abejas según el hábitat donde se recolectaron mediante un ANOVA un Factor, datos agrupados, usando G-Stat 2.0. Finalmente, se hizo un análisis de similitud para las especies de euglosinos colectadas según el tipo de hábitat, por el método de Chi-cuadrado (WPGMA) usando MVSP $3.13 \mathrm{~m}$.

\section{RESULTADOS}

Se capturaron 17 especies de la tribu Euglossini, de 26 conocidas para los municipios muestreados (Parra-H y Nates-Parra 2004) representadas por tres géneros: Euglossa Latreille 1802 (10 especies), Eulaema Lepeletier 1841 (cinco especies) y Exaerete Hoffmannsegg 1817 (dos especies). Fueron muestreadas en total 19 localidades: cuatro en Acacías, cinco en Cumaral y 10 en Villavicencio. Se encontraron diferencias significativas para la comunidad de abejas de las orquídeas según el tipo de paisaje muestreados $(\mathrm{F}=11.7484, \mathrm{p}<0.0001, \alpha=0.05)$. Se estableció la dispersión de las especies por localidad (Fig. 1) además del grado de similitud para las especies colectadas según el hábitat muestreado (Fig. 2).

Si bien el número de especies colectadas no es muy distinto para Villavicencio y Acacías, la diversidad fue mayor para este primer municipio, ya que algunas localidades presentaron una ponderable frecuencia de especies. La diversidad para localidades en Villavicencio fue $\mathrm{H}=2.68803209$ (12 especies), Acacías $\mathrm{H}=1.70205999$ (13 especies) y Cumaral $\mathrm{H}=1.07873118$ (siete especies). Las localidades con mayor diversidad fueron: La colonia penal (Municipio de Acacías), Caño el Buque, El Jardín Botánico, Barrio la Coralina, La Corocora (Municipio de Villavicencio) y San Nicolás (Municipio de
Cumaral) (ver Cuadro 1. Nótese el tipo de hábitat al que corresponden las localidades).

Según el tipo de paisaje se registran las siguientes especies de euglosinos: especies exclusivas de ambientes conservados: Euglossa cybelia Moure 1968, Eulaema speciosa Mocsáry 1897 y Exaerete frontalis Guérin-Méneville 1845. Sólo se registra a Euglossa deceptrix Moure 1968 y E. hemichlora Cockerell 1917 como especies exclusivas de ambientes urbanos y rurales respectivamente. E. heterosticta Moure 1968 se registró tanto en ambientes urbanos como conservados. Exaerete smaragdina Guérin-Méneville 1845 se colectó únicamente en ambientes urbanos y rurales. Euglossa magnipes Dressler 1982, E. singularis Mocsáry 1899, E. chalyeata Friese 1925 y Eulaema bombiformis Packard 1869 fueron especies colectadas tanto en paisajes conservados como rurales. Las especies restantes: Euglossa imperialis Cockerell 1922, E. modestior Dressler 1982, E. tridentata Moure 1970, Eulaema cingulata Fabricius 1804 y E. nigrita Lepeletier 1841 fueron comunes en todos los tres tipos de paisajes muestreados (Fig. 3).

Respecto a la distribución de especies por localidad E. nigrita fue la especie más ubicua en las localidades muestreadas, mientras que ocurrieron especies exclusivas para varias localidades como E. cybelia, E. speciosa y E. frontalis (Colonia Penal), E. deceptrix (Barrio La Coralina) y E. hemichlora (San Nicolás). Se registraron especies en solo dos localidades

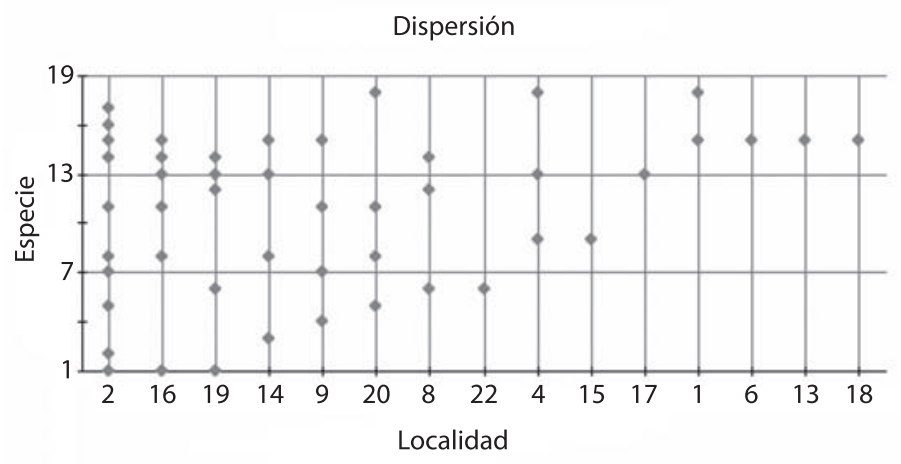

Fig. 1. Dispersión de datos, localidad según especie.

Fig. 1. Data dispersion, locality against species. 


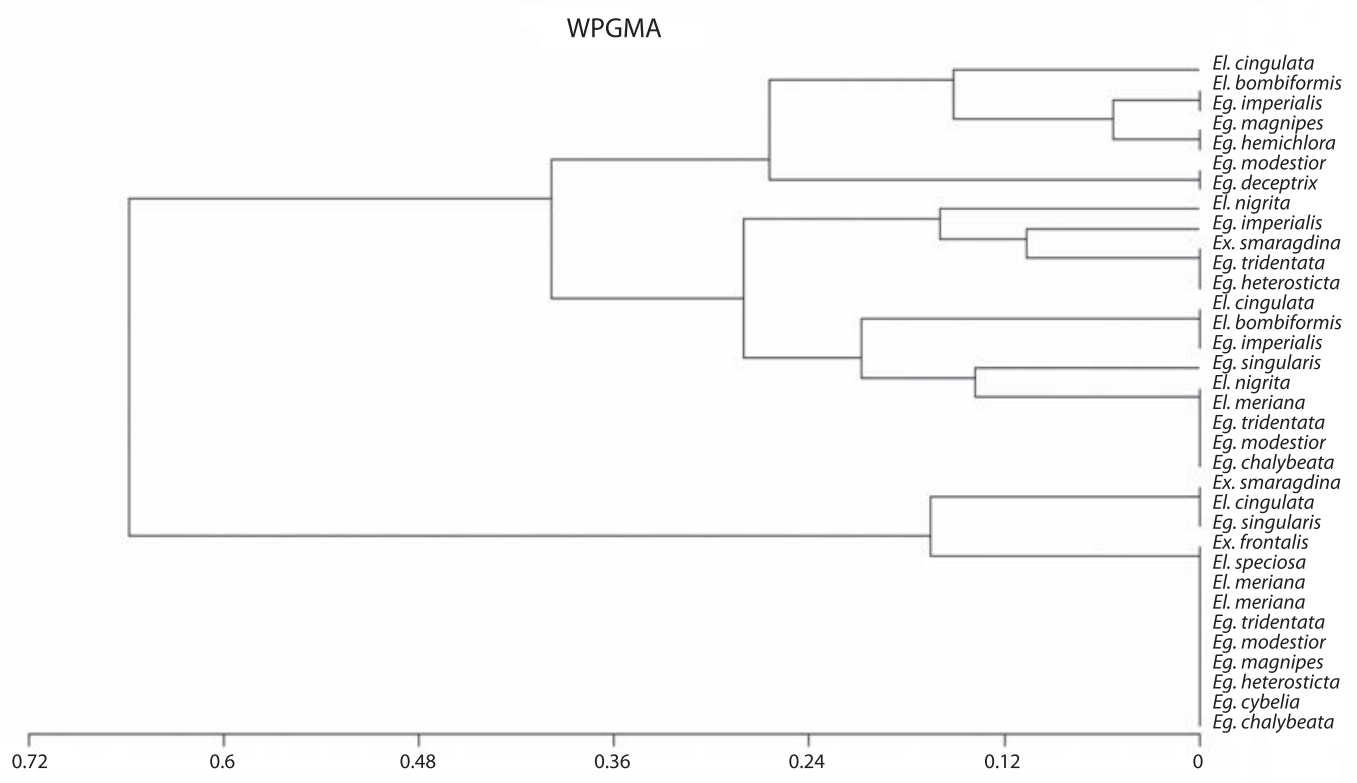

Chi-Squared

Fig. 2. Agrupamiento de similitud Chi-cuadrado para las especies de abejas euglosinas según tipo de hábitat de los municipios muestreados (WPGMA).

Fig. 2. Chi-squared cluster for euglossine bee species by habitat type in the sampled areas (WPGMA).

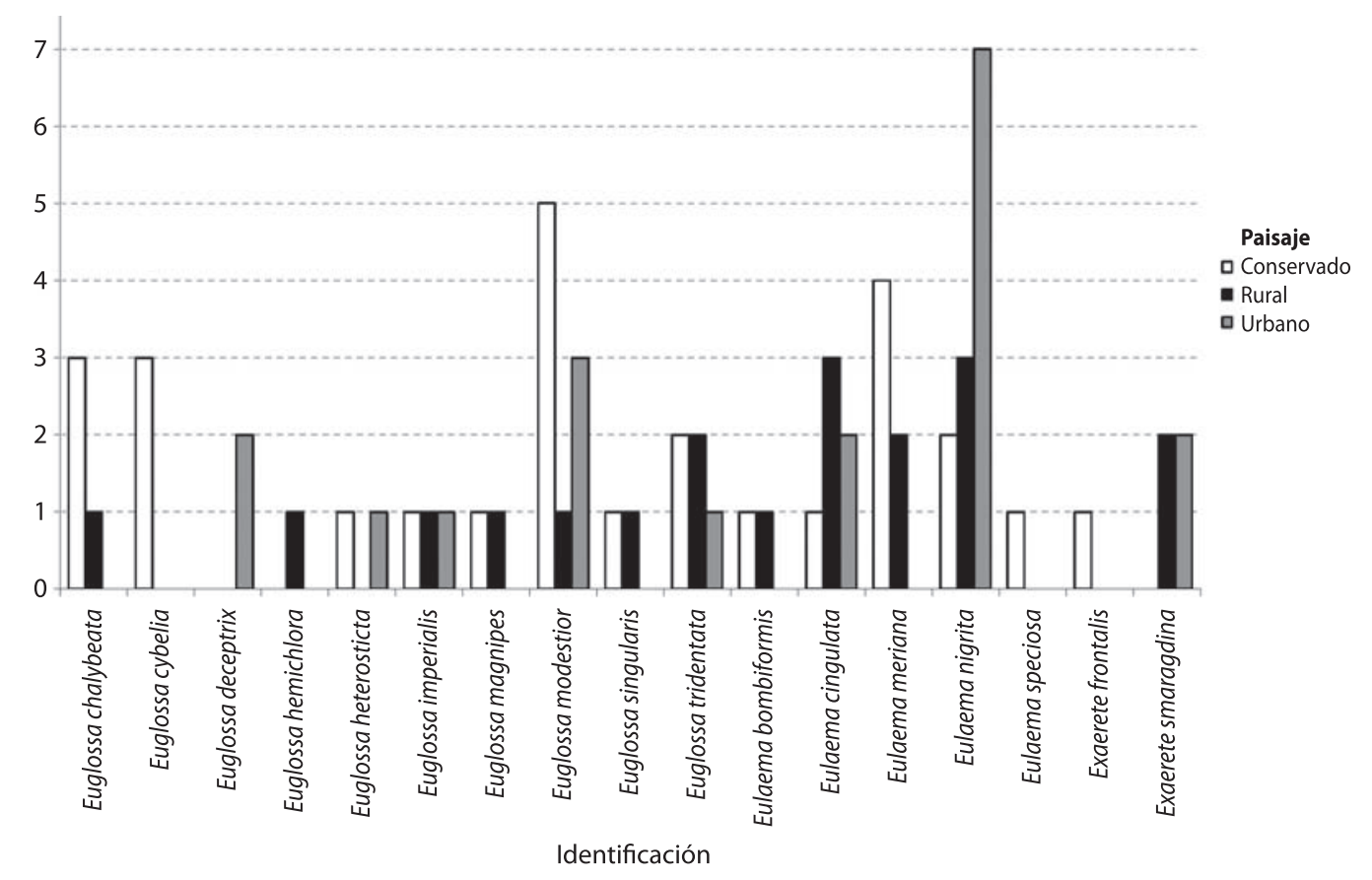

Fig. 3. Especies de euglosinos por paisaje para los municipios muestreados.

Fig. 3. Euglossine bee species by habitat in the sampled areas. 
CUADRO 1

Códigos para especie, localidad y municipio

TABLE 1

Codes for species, locality and municipal division

Identificación

Euglossa chalybeata

Euglossa chalybeata

Euglossa chalybeata

Euglossa chalybeata

Euglossa cybelia

Euglossa deceptrix

Euglossa hemichlora

Euglossa heterosticta

Euglossa heterosticta

Euglossa imperialis

Euglossa imperialis

Euglossa imperialis

Euglossa magnipes

Euglossa magnipes

Euglossa modestior

Euglossa modestior

Euglossa modestior

Euglossa modestior

Euglossa singularis

Euglossa singularis

Euglossa tridentata

Euglossa tridentata

Euglossa tridentata

Euglossa tridentata

Eulaema bombiformis

Eulaema bombiformis

Eulaema cingulata

Eulaema cingulata

Eulaema cingulata

Eulaema cingulata

Eulaema cingulata

Eulaema meriana

Eulaema meriana

Eulaema meriana

$$
\text { Municipio }
$$

1 Acacías

1 Acacías

1 Villavicencio

1 Villavicencio

2 Acacías

3 Villavicencio

4 Cumaral

5 Acacías

5 Villavicencio

6 Cumaral

6 Villavicencio

6 Villavicencio

7 Acacías

7 Cumaral

8 Acacías

8 Villavicencio

8 Villavicencio

8 Villavicencio

9 Acacías

9 Villavicencio

11 Acacías

11 Cumaral

11 Villavicencio

11 Villavicencio

12 Cumaral

12 Villavicencio

13 Acacías

13 Villavicencio

13 Villavicencio

13 Villavicencio

13 Villavicencio

14 Acacías

14 Cumaral

14 Villavicencio
Localidad

1 Colonia penal*

1 Colonia penal. Las Blancas*

4 Caño el Buque**

4 Jardín Botánico*

1 Colonia penal*

4 Barrio La Coralina***

2 Vía San Nicolás**

1 Colonia Penal. Las Blancas*

4 La Corocora***

2 Finca Los Arrayanes**

4 Jardín Botánico*

4 Pozo azul***

1 Colonia Penal. Las Blancas*

2 Vía San Nicolás**

1 Colonia Penal. Las Blancas*

4 Barrio La Coralina***

4 Caño el Buque**

4 La Corocora***

1 Vereda San José**

4 Buenavista*

1 Colonia Penal. Las Blancas*

2 Vía San Nicolás**

4 Caño el Buque**

4 La Corocora***

2 Finca los Arrayanes**

4 Jardín Botánico*

1 Vereda San José**

4 Barrio La Coralina***

4 Caño el Buque**

4 E.B.T.R.F***

4 Jardín Botánico*

1 Colonia penal. Las Blancas*

2 Finca los Arrayanes**

4 Caño el Buque** 
CUADRO 1 (Continuación)

Códigos para especie, localidad y municipio

TABLE 1 (Continued)

Codes for species, locality and municipal division

Identificación

Eulaema meriana

Eulaema nigrita

Eulaema nigrita

Eulaema nigrita

Eulaema nigrita

Eulaema nigrita

Eulaema nigrita

Eulaema nigrita

Eulaema nigrita

Eulaema speciosa

Exaerete frontalis

Exaerete smaragdina

Exaerete smaragdina

Exaerete smaragdina
Municipio

14 Villavicencio

15 Acacías

15 Acacías

15 Cumaral

15 Cumaral

15 Villavicencio

15 Villavicencio

15 Villavicencio

15 Villavicencio

16 Acacías

17 Acacías

18 Acacías

18 Acacías

18 Villavicencio
Localidad

4 Jardín Botánico**

1 Acacías***

1 Colonia penal*

2 Cementerio***

2 Vía San Nicolás**

4 Barrio El Porvenir*** 13

4 Barrio La Coralina*** 14

4 Caño el Buque** 16

4 El Barzal*** 18

1 Colonia penal* 2

1 Colonia penal* 2

1 Acacías*** 1

1 Vereda San José** 4

4 La Corocora*** 20

Corresponde para las localidades: Conservado*, Rural** y Urbano***

como E. imperialis (Finca los arrayanes y único registro para Pozo Azul), E. magnipes (San Nicolás y Colonia Penal), E. heterosticta (La Corocora y Colonia Penal), E. bombiformis (Jardín Botánico y Finca Los Arrayanes) y $E$. singularis (Vereda San José y registro único para Buenavista). E. cingulata ocurrió de manera exclusiva en el Estación de Biología Tropical Roberto Franco (Villavicencio, urbano) mientras E. nigrita fue exclusiva para Cementerio (Cumaral, urbano), Barrio el Porvenir y El Barzal (Villavicencio, urbano). Sin embargo estas especies fueron comunes para muchas localidades y tipos de paisaje (Fig. 1).

Según el análisis de similitud para las especies de euglosinos según el tipo de hábitat muestreado, las especies registradas en hábitats conservados se agrupan con cerca del 12 $\%$ de similitud, en general se presenta una alta semejanza y no se observan agrupamientos claros (Fig. 2).

\section{DISCUSIÓN}

Este trabajo puede interpretarse como una aproximación metodológica que permita valorar la calidad del hábitat en el que se encuentran especies euglosinas dadas, asumiendo que debe tenerse un conocimiento previo de la biología de dichas especies: Para esto hay que considerar que la interpretación de los muestreos de abejas euglosinas presenta muchas dificultades. Algunas de éstas dificultadas están dadas por las distintas preferencias de los machos por aromas, siendo algunas especies no atraídas por ninguno y otras atraídas por compuestos muy específicos (Dodson et al. 1969, Nemésio y Silveira 2004). La abundancia de machos atraídos a un cebo depende de las condiciones de humedad y temperatura del hábitat muestreado por lo cual no es una referencia confiable en cuanto a la abundancia de ciertas especies respecta (Dressler 1969). 
Sin embargo la preferencia de los machos por los distintos tipos de aroma no varía intraespecíficamente por lo cual la diversidad especies de abejas de las orquídeas en un hábitat dado, es representativa y significativa para el cebo empleado (Dressler 1969). En este trabajo, se emplean índices de diversidad basados en la frecuencia en que las especies ocurrieron en las distintas localidades, por lo cual la correspondencia entre la distribución de las especies de euglosinos y el paisaje donde se capturaron es confiable. Cabe anotar que en este trabajo se encontró de manera desbalanceada los tipos de hábitat por municipio, error que se disminuyó usando una prueba estadística con métodos de agrupamiento.

Considerando que los cambios en un hábitat afectan de manera más evidente a especies ápteras, ya sea en algún estadio de su ciclo de vida (Magagula 2003), es de esperar que interpretar la calidad de distintos paisajes dado el grado de deterioro por presión antrópica, usando organismos de vuelo largo es una tarea difícil. La presencia de las distintas especies de abejas de las orquídeas y su relación con la calidad del medio, en los diferentes paisajes está relacionada con varias características biológicas (Dressler 1982). Entre estas, hay que considerar que en muchas especies de euglosinos, habita alguna variación en la longitud de sus lenguas y tamaños corporales. Este hecho restringiría algunas especies a recursos vegetales específicos, presentando una marcada preferencia por néctares más fluidos de familias de plantas particulares, con morfologías florales típicas (Ackerman 1985, Kato et al. 1992) donde es favorecida la succión de este tipo de néctares, permitiendo la obtención de energía más eficientemente y así suplir las necesidades típicas de organismos con alta potencia de vuelo (Kroodsma 1975, Dudley 1995, Borrel 2004). También es importante considerar que las abejas de gran tamaño pueden regular la temperatura corporal aumentando el flujo de hemolinfa del tórax al abdomen (Inouye 1975, Borrell y Medeiros 2004) lo cual les permite incursionar en ambientes que presenta una menor humedad frente a una mayor temperatura, ya sea estratos altos del dosel o áreas abiertas yuxtapuestas a cobertura vegetal (Otero y Sandino 2003). De esta forma existen especies que por la longitud de su lengua y tamaño corporal, no serían aptas para "colonizar" áreas abiertas o donde el recurso se restringe a plantas con corolas profundas y/o cerca de los estratos altos del dosel. Sin embargo como afirman Otero y Sandino (2003) algunas de estas especies (incluyendo las pequeñas), podrían ser oportunistas frente a recursos para el cual pueden acceder entrando en la flor o explotando otras fuentes energéticas más abundantes como las ofrecidas en borde de bosque (Powell y Powell 1987).

De igual forma, las abejas euglosinas exhiben distintos grados de sociabilidad, que favorecería la explotación de recursos escasos en áreas deterioradas, restringiendo a aquellas especies menos cooperativas a lugares donde el recurso es óptimo para la supervivencia (Parra et al 2005). Por último los machos son territoriales (Kimsey 1980) y presentan una estrategia de forrageo donde las abejas cubren largas distancias en busca de los recursos dispersos en el bosque, realizando paradas periódicas (Trap linning).

Con estas consideraciones mencionadas anteriormente, la diversidad relativa sería proporcional a la calidad del medio, cuando habita la máxima variación de formas y tamaños dentro de la tribu, ya que por ende se está considerando que existen recursos lo suficientemente variados y disponibles (dentro el espectro de oligolectia de los euglosinos) para mantener las especies representadas en las muestra. Además de esto se consideraron abejas capturadas en cebos muy generalistas o explotando algún recurso vegetal lo cual permite afirmar que la muestra representa una amplitud de nicho ponderable para las interacciones que estos dos indicadores representan, es decir, que incluyen ámbitos primordiales de la biología de las especies de este grupo de abejas.

La presencia de especies parásitas estaría relacionada solo con la presencia del hospedero. Así E. smaragdina presenta una distribución similar a $E$. nigrita mientras $E$. frontalis se distribuiría de manera parecida a especies como $E$. 
meriana (o E. speciosa). Localidades como la Colonia Penal Agropecuaria (Acacías) y Jardín Botánico (Villavicencio) son ambientes que presentan una calidad buena en cuanto oferta de recursos para machos y hembras. Por otro lado, la composición y cercanía de los fragmentos de bosque en la vereda San José en Acacías sería un factor que mejoraría las condiciones para algunas especies en ambientes perturbados ya que los fragmentos se constituirían como un paisaje más o menos homogéneo y no como unidades aisladas.

Aunque la diferencia en diversidad de especies de abejas de las orquídeas en los distintos paisajes no presenta grandes diferencias (conservado: 14 especies, rural: 12 especies y urbano: 8 especies) en áreas rurales y urbanas se observa la presencia de pocos tipos de especies que se pueden suponer como plásticas como E. nigrita y E. cingulata (Fig. 3) que toleran de manera más eficiente el disturbio y la contaminación, incursionando en localidades que ofrecen los recursos estrictamente necesarios, como lo son las áreas urbanas (Alysson et al. 2005, Parra-H et al. 2005). Las áreas rurales y cascos urbanos con cobertura vegetal contigua, por presentar focos de oferta de recursos, permitirían la instauración y mantenimiento de aquellas especies no facultativas o tolerantes del disturbio (por ejemplo San Nicolás para $E$. magnipes). Por lo general se puede decir que la mayoría de áreas urbanas y rurales cuando no presentan dichas coberturas son localidades con una regular a mala calidad según la oferta de recursos (Cuadro 2).

Dressler (1969) sugiere que con estos muestreos y con este tratamiento para la diversidad de especies de euglosinos, los agrupamientos de especies se pueden considerar homogéneos y por lo tanto suponer sobrelapamientos entre localidades. Sin embargo para este trabajo esto no es válido debido a las diferencias biológicas y ecológicas que consideramos y por el hecho que asumimos a los tipos de paisaje, como una variable en la distribución de especies. Es posible que definiendo niveles de interacción y frente a características físicas de un hábitat dado (tipos de vegetación más detallados), se obtenga, para los índices de diversidad usados para comunidades de abejas de las orquídeas, estimativos numéricos o cuantitativos que expliquen con mayor precisión la calidad del medio, basado en estas interacciones y según el número y tipo de especies. A la vez, al realizar estos estimadores se obtendrán datos exclusivos para las localidades de la región muestreada, constituyendo modelos que permitirán establecer estrategias, que junto con monitoreos en regiones críticas, permitan formular medidas de conservación.

Cabe anotar, como lo reveló el análisis de similitud de especies para los tipos de paisajes muestreados, que la frecuencia de ambientes con un grado de conservación aceptable es muy baja y que las diferencias entre ambientes urbanos y rurales no es significativa. Esto habita

\section{CUADRO 2}

Frecuencia de especies de euglosinos por tipo de paisajes para las localidades muestreadas

TABLE 2

Euglossine species frequency by landscape type for the sampled municipalities

$\begin{array}{lccc}\text { Especie } & \text { Conservado } & \text { Rural } & \text { Urbano } \\ \text { Euglossa chalybeata } & 10.7 & 5.3 & - \\ \text { Euglossa cybelia } & 10.7 & - & - \\ \text { Euglossa deceptrix } & - & - & 10.5 \\ \text { Euglossa hemichlora } & - & 5.3 & - \\ \text { Euglossa heterosticta } & 3.6 & - & 5.3 \\ \text { Euglossa imperialis } & 3.6 & 5.3 & 5.3 \\ \text { Euglossa magnipes } & 3.6 & 5.3 & - \\ \text { Euglossa modestior } & 17.9 & 5.3 & 15.8 \\ \text { Euglossa singularis } & 3.6 & 5.3 & - \\ \text { Euglossa tridentata } & 7.1 & 10.5 & 5.3 \\ \text { Eulaema bombiformis } & 3.6 & 5.3 & - \\ \text { Eulaema cingulata } & 3.6 & 15.8 & 10.5 \\ \text { Eulaema meriana } & 14.3 & 10.5 & - \\ \text { Eulaema nigrita } & 7.1 & 15.8 & 36.8 \\ \text { Eulaema speciosa } & 3.6 & - & - \\ \text { Exaerete frontalis } & 3.6 & - & - \\ \text { Exaerete smaragdina } & - & 10.5 & 10.5\end{array}$


por que en esta región del país las prácticas ganaderas han disminuido considerablemente la extensión de áreas con cobertura vegetal nativa (Madriñán 2001, Márquez 2001, ParraH y Nates-Parra 2004). Este hecho se refleja en que no hay un agrupamiento claro, de manera general, de las especies colectadas aunque como se mencionó anteriormente, todas aquellas especies exclusivas de ambientes conservados se agrupan con cerca del $12 \%$ de similitud. Además abejas del género Eufriesea Cockerell 1908 no fueron colectadas durante los muestreos a pesar que estos incluyeron ambos ciclos estacionales en el piedemonte llanero.

De las especies registradas en este trabajo, la mayoría son reportadas por otros autores asociadas también a áreas conservadas como reservas forestales: E. cybelia, E. heterosticta (Jansen et al. 1981), E. singularis (Dressler 1985), E. bombiformis (Jansen et al. 1981, Powell y Powell 1985, Dressler 1985) y E. frontalis (Jansen et al. 1981, Powell y Powell 1985). Este conjunto de especies, además de E. magnipes (registrada en este trabajo), pueden considerase como especies exclusivas de ambientes en aceptable a buen estado de conservación (áreas conservadas o rurales con coberturas vegetales importantes) ya que dichas especies ocurrieron el lugares donde la diversidad en composición tamaños y formas biológicas en general fue alta y no ocurrieron en lugares donde el deterioro del medio era evidente.

La ecología de poblaciones de abejas de las orquídeas resulta difícil, tanto metodológicamente como interpretativamente, por lo cual es importante reunir la mayor cantidad de información sobre la biología básica y comportamiento de este grupo de insectos (Parra-H y OspinaTorres, pers. obs). A pesar de esto, la metodología e interpretación propuesta en este trabajo, en adición a las consideraciones biológicas de los euglosinos, podría permitir dilucidar cambios o tendencias sustanciales del medio, dado el comportamiento en la distribución de las especies más sensibles y también las más plásticas.
Permitiría generar métodos de monitoreo para regiones tanto alteradas como naturales.

\section{AGRADECIMIENTOS}

Los autores agradecen a los integrantes de LABUN (A. Rodríguez, P. Baquero, D. Vélez, M. Gómez, B. Mantilla, E. Palacios y J. Rosso), a Mariana Cadavid por la revisión del manuscrito y Rodulfo Ospina-Torres por sus valiosos comentarios, a la dirección de la Colonia Penal Agropecuaria del Municipio de Acacías, comunidad Vereda San José de Acacías, a la Estación de Biología Tropical Roberto Franco (EBTRF) por el apoyo logístico, a la Facultad de Ciencias, Departamento de Biología, Universidad Nacional de Colombia Sede Bogotá y a la División de Investigaciones de la sede Bogotá DIB (convocatoria Prometeo No. 20101002454 U.N.) por la financiación de este trabajo.

\section{RESUMEN}

Las abejas de las orquídeas subsisten en vastas áreas de bosque tropicales gracias a que mantienen estrechas relaciones con tipos de vegetación particular en diversos micro-hábitats. Con base en este tipo de relaciones con el medio y características biológicas como preferencia por ciertos tipos de néctares y de polen, y diversidad morfológica y etológica de la tribu, es posible evaluar la calidad de un hábitat según la distribución de euglosinos. Este trabajo propone el uso de esta información, además de índices de diversidad para la evaluación de la calidad del medio. Entre marzo y diciembre de 2003 muestreamos tres tipos de paisaje (Urbano, Rural y Conservado) en el piedemonte llanero colombiano, usando redes entomológicas y sustancias aromáticas (Cineol y Metil Salicilato). Para las 15 localidades muestreadas se registraron 17 de las 26 especies conocidas para el área. Eulaema nigrita fue la más frecuente mientras que Euglossa magnipes, E. cybelia, E. heterosticta, E. singularis, Eulaema bombiformis, E. speciosa y Exaerete frontalis correspondieron a ambientes considerados de aceptable a buena calidad. La composición y cercanía de fragmentos de bosque son factores favorables. La diversidad relativa (máxima variación de formas y tamaños dentro de la tribu), sería proporcional a la calidad del medio. 
Palabras clave: comunidades, conservación, diversidad, euglosinos, piedemonte llanero colombiano.

\section{REFERENCIAS}

Ackerman, J.D. 1985. Euglossine bees and their nectar hosts, p. 225-233. In W.G. D'Arcy \& M.D. Correa A. (eds.). The botany and natural history of Panama. Missouri Botanical Garden, San Luis, Misurí, EEUU.

Alysson, K., P. de Sousa, I. Malva, M. Hernández \& C.F. Martins. 2005. Riqueza, abundância e diversidade de Euglossina (Hymenoptera, Apidae) em três áreas da Reserva Biológica Guaribas, Paraíba, Brasil. Rev. Bra. Zool. 22: 320-325.

Blydenstein, J. 1967. The savanna vegetation of the llanos of Colombia. Ecology 48: 1-15.

Bonilla-Gómez, M.A. \& G. Nates-Parra. 1992. Abejas euglosinas de Colombia (Hymenoptera: Apidae) I. Claves ilustradas. Caldasia 17: 149-172.

Bonilla-Gómez, M.A. 1997. Uso de las abejas euglosinas para monitoreo de la diversidad en áreas de conservación. Tacayá 7: 2-7.

Borrell, B. \& M.J. Medeiros. 2004. Thermal stability and muscle efficiency in hovering orchid bees (Apidae: Euglossini). J. Exp. Biol. 207: 2 925-2 933.

Borrell, B. 2004. Suction feeding in orchid bees (Apidae: Euglossini). Proc. R. Soc. Lond. B. 271: 164-166.

Cameron, S.A. 2004. Phylogeny and biology of neotropical orchid bees (Euglossini). Annu. Rev. Entomol. 2004. 49: 377-404.

CORPOMACARENA. 2004. Plan de acción trienal 20042006. Corporación para el desarrollo sostenible del área de manejo especial de la Macarena, Villavicencio, Colombia. Macarena, Villavicencio, Colombia-

Dodson, C.H., R.L. Dressler, H.G. Hills, R.M. Adams \& N.H. Williams. 1969. Biologically active compounds in orchid fragrances. Science 164: 1 243-1 249.

Dressler, R.L. 1968. Pollination by euglossine bees. Evolution 22: 202-210.

Dressler, R.L. 1969. Species diversity of Euglossa in Panama. Ecology 50: 713-716.

Dressler, R.L. 1982. Biology of the orchid bees (Euglossini). Ann. Rev. Ecol. Syst. 13: 373-394.
Dressler, R.L. 1985. Euglosine bees (Hymenoptera: Apidae) of the Tambopata Reserved Zone, Madre de Dios, Perú. Rev. Per. Ent. 27: 75-79.

Dudley, R. 1995. Extraordinary flight performance of orchid bees (Apidae: Euglossini) hovering in heliox (80\% He/20 \% O ). J. Exp. Biol. 198: 1 065-1 070.

Inouye, D.W. 1975. Flight temperatures of male euglosine bees (Hymenoptera: Apidae: Euglossini). J. Kansas. Entomol. Soc. 48: 366-370.

Jansen, D.H., P.J. De Vries, M.L. Higgins \& L.S. Kimsey. 1981. Seasonal and site variation in Costa Rican euglossine bees at chemical baits in lowland deciduous and evergreen forests. Ecology 63: 66- 74.

Kato, M., D.W. Roubik. \& I. Inoue. 1992. Foraging behavior and concentration preferences of male Euglossine bees (Hymenoptera: Apidae). Tropics 1: 259-264.

Kimsey, L.S. 1980. The behavior of male orchid bees and the question of leks. Anim. Behav. 28: 996-1 004.

Kroodsma, D.E. 1975. Flight Distances of Male Euglossine Bees in Orchid Pollination. Biotropica 7: 71-72.

Macarthur, R.H. \& J. Macarthur. 1961. On bird species diversity. Ecology 42: 594-598.

Madriñan, L.F. 2001. Proceso de transformación de un bosque aluvial del piedemonte llanero y su impacto sobre la vegetación (Puerto López-Meta, 1939-1997). Trabajo de Grado para optar al Título de Ecólogo, Pontificia Universidad Javeriana, Facultad de Estudios Ambientales y Rurales, Bogotá, Colombia.

Magagula, C.N. 2003. Changes in carabid beetle diversity within a fragmented agricultural landscape. Afr. J. Ecol. 47: 23-30.

Márquez, G. 2001. De la abundancia a la escasez. La transformación de ecosistemas en Colombia, p. 186. In G. Palacios (ed.). La naturaleza en disputa. Universidad Nacional de Colombia, Unibiblos, Bogotá, Colombia.

Nemésio, N. \& F.A. Silveria. 2004. Biogeographic notes on rare species of Euglossina (Hymenoptera: Apidae: Apini) occurring in the brazilian atlantic rain forest. Neotrop. Entomol. 33: 117-120.

Otero, T.J. \& J.C. Sandino. 2003. Capture rates of male euglossine bees across a human intervention gradient, Chocó region, Colombia. Biotropica 65: 520-529.

Parra-H, A. \& G. Nates-Parra. 2004. Abejas de las orquídeas del piedemonte llanero, p. 74-77. In G. Nates- 
Parra. \& M.I. Gómez. (eds.). Libro de Memorias. II Encuentro Colombiano sobre Abejas Silvestres, Bogotá, Colombia.

Parra-H, A., G. Nates-Parra. \& M. Cadavid. 2005. Abejas de las orquídeas en dos ambientes con distinta perturbación en el piedemonte llanero. ¿Es la estructura social una ventaja o un límite de supervivencia?, p. 127. In P. Chacón \& M. Chaves. (eds.). Libro de Resúmenes. V Coloquio de Insectos Sociales IUSSI Sección Bolivariana. Universidad del Valle, Facultad de Ciencias, Cali, Colombia.

Powell, A.H. \& G.V.N. Powell. 1987. Population dynamics of male euglossine bess in amazonian forest fragment. Biotropica 19: 176-179.

Ramírez, S., R.L. Dressler \& M. Ospina. 2002 Abejas euglosinas (Hymenoptera: Apidae) de la región neotropical. Biota Colombiana 3: 7-118.

Rangel-Ch., J.O. \& M. Aguilar-P. 1995. Una aproximación sobre la diversidad climática en las regiones naturales de Colombia, p 25-76. In J.O. Rangel-Ch. (ed.). Colombia diversidad biótica I. Instituto de Ciencias Naturales. Universidad Nacional de Colombia, Bogotá. Colombia.

Rodríguez-C.Á. 2005. Forrajeo de polen por obreras de Melipona fasciata (Hymenoptera: Apidae: Meliponini) en una zona rural del piedemonte llanero (Acaías-Meta-Colombia). Trabajo de Grado para optar al título de Biólogo, Universidad Nacional de Colombia, Facultad de Ciencias, Bogotá, Colombia.

Roubik, D.W. \& P.E. Hanson. 2004. Orchid bees of tropical America: Biology and field guide. INBIO, Heredia, Costa Rica.

Roubik, D.W. 1989. Ecology and natural history of tropical bees. Cambridge University, Cambridge, Inglaterra.

Silva, F.S. \& J.M.M. Rebêlo. 2002. Population dynamics of euglossine bees in an early second-growth forest of Cajual island, in the state of Maranhão, Brazil. Braz. J. Biol. 62: 15-23. 
